\title{
Educação Interprofissional em Saúde: Revisão Integrativa da Literatura Brasileira (2008-2018)
}

Interprofessional Education in Healthcare: Integrative Review of Brazilian Literature (2008-2018)

\section{Educación Interprofesional en Salud: Revisión Integradora de la Literatura Brasileña} (2008-2018)

\author{
Ana Paula de Sousa Silva Baquião ${ }^{1}$ \\ Bruna Costa de Almeida \\ Leila Garcia Ribeiro Silva \\ Universidade Federal de Juiz de Fora (UFJF) \\ Rodrigo Sanches Peres \\ Universidade Federal de Uberlândia (UFU) \\ Fabiane Rossi dos Santos Grincenkov \\ Universidade Federal de Juiz de Fora (UFJF)
}

\begin{abstract}
Resumo
Este estudo teve como objetivo estabelecer um panorama da produção científica nacional relativa à Educação Interprofissional em Saúde (EIP), com ênfase nas pesquisas desenvolvidas junto a pósgraduandos ou profissionais de saúde ao longo de uma década (2008-2018). Trata-se de uma revisão integrativa realizada mediante consultas às bases de dados MEDLINE, LILACS, SciELO-Brasil e Scopus. Compuseram o corpus de análise 31 referências, as quais foram examinadas segundo um conjunto de dimensões analíticas. Evidenciou-se que as referências se concentram nos últimos cinco anos e, predominantemente, foram assinadas por pesquisadores vinculados a universidades públicas, derivam de pesquisas qualitativas voltadas à prática interprofissional e tiveram como participantes profissionais de saúde inseridos em serviços de atenção primária. Conclui-se que o fortalecimento da EIP no país se afigura como um importante caminho a ser percorrido no sentido da integralidade das ações e dos serviços de saúde, e que novas pesquisas são necessárias para tanto.

Palavras-chave: profissionais da saúde, formação dos profissionais de saúde, revisão de literatura
\end{abstract}

\begin{abstract}
This study aimed to establish an overview of the Brazilian scientific production related to Interprofessional Education in Healthcare (IPE), emphasizing researches developed with graduate students or healthcare personnel participation along a decade (2008-2018). This is an integrative review carried out in MEDLINE, LILACS, SciELO-Brasil and Scopus databases. The corpus of analysis consisted of 31 references, which were examined according to a set of analytical dimensions. It was evidenced that the references are concentrated in the last five years, and, predominantly, were signed by researchers from public universities, derive from qualitative research focused on interprofessional practice, and had as participants professionals inserted in primary healthcare services. It can be concluded that the strengthening of IPE in Brazil is an important way towards the integrality of health actions and services, and that new research is needed for this purpose.

Keywords: postgraduate training, health professionals, health professionals' education, literature
\end{abstract} review

\section{Resumen}

Este estudio tuvo como objetivo establecer una visión general de la producción científica brasileña relacionada con la Educación Interprofesional en Salud (EIP), con énfasis en investigaciones realizadas con la participación de estudiantes de posgrado o profesionales de salud a lo largo de una década. Esta es una revisión integradora realizada a través de consultas a las bases de datos MEDLINE, LILACS, SciELO-Brasil y Scopus. El corpus de análisis comprendió 31 referencias, que fueron examinadas de acuerdo con un conjunto de dimensiones analíticas. Se evidenció que las referencias se concentraron

\footnotetext{
${ }^{1}$ Endereço para contato: Rua José Lourenço Kelmer, s/n, São Pedro, Juiz de Fora, MG, Brasil, CEP 36036-900. E-mail: anapaulasousa.psico@gmail.com
} 
en los últimos cinco años, y, predominantemente, fueron firmadas por investigadores vinculados a universidades públicas, son derivadas de investigaciones cualitativas centradas en la práctica interprofesional y tuvieran como participantes profesionales de salud de servicios de atención primaria. Se puede concluir que el fortalecimiento de la IPE en Brasil es una forma importante de avanzar hacia la integralidad de las acciones y servicios de salud, y que se necesita más investigaciones para este fin.

Palabras clave: posgrado, profesionales de la salud, formación de los profesionales de la salud, revisión de literatura

\section{Introdução}

A Educação Interprofissional em Saúde (EIP) tem ocupado um lugar de crescente destaque nas propostas de aperfeiçoamento das políticas públicas de saúde em diversos países (Reeves, 2016). Porém, os primeiros debates acerca da relevância do trabalho em equipe para a reorientação da formação e da prática em saúde surgiram há décadas, já que movimentos pioneiros nesse sentido se sucederam em serviços de saúde do Reino Unido nos anos 1960 (Barr, 2015). Na América, segundo Blue, Brandt e Schimitt (2010), a colaboração entre diferentes profissões do campo da saúde começou a se intensificar a partir da década de 1970, sobretudo em centros de saúde vinculados a universidades estadunidenses. A despeito disso, comumente atribui-se a origem do conceito de EIP a um grupo de especialistas que, em 1986, se reuniu em um evento promovido pela Organização Mundial de Saúde (OMS) (Kantrowitz, Kaufman, Mennin, Fülöp, \& Guilbert, 1987).

$\mathrm{Na}$ atualidade, algumas entidades têm exercido um importante papel na difusão da EIP no mundo. O Centre for the Advancement of Interprofessional Education (CAIPE) é uma delas, na medida em que, em 2013, publicou um guia, intitulado Introducing Interprofessional Education (Barr \& Low, 2013), com diversas orientações para a operacionalização da EIP, inclusive com indicações sobre a abordagem do tema na graduação e na pós-graduação. Já - Canadian Interprofessional Health Collaborative $(\mathrm{CICH})$ disponibiliza, em seu website, uma biblioteca virtual composta por publicações voltadas ao compartilhamento de experiências exitosas e à ampliação de discussões relativas à EIP (Reeves, Abramivich \& Goldman, 2009). Como reflexo do trabalho desenvolvido por autores associados às referidas entidades ou a outras que têm se ocupado da EIP, mostrou-se inegável seu valor como estratégia capaz de elevar a qualidade da atenção em saúde. Afinal, compreende-se que a EIP ocorre quando, por meio de práticas colaborativas, membros de duas ou mais profissões do campo da saúde - estudantes ou profissionais - aprendem e trabalham em conjunto, visando à melhoria do cuidado oferecido aos pacientes (Barr \& Low, 2013; Canadian Interprofessional Health Collaborative [CIHC], 2010; OMS, 2010).

No que diz respeito especificamente à realidade nacional, Feuerwerker e Capozzolo (2013) apontam que as primeiras críticas ao caráter uniprofissional e tecnicista dos modelos de formação e atuação dos profissionais de saúde no Brasil - consideradas um ponto de partida da EIP - surgiram timidamente nas décadas de 1960 e 1970, sobretudo com iniciativas isoladas no âmbito acadêmico. Todavia, foi em 1988, com o advento da Constituição da República Federativa do Brasil e a consequente implementação do Sistema Único de Saúde (SUS), que a saúde pública no país passou a ser norteada por uma concepção ampliada de saúde, a qual pressupõe a integração de saberes e práticas. Já o alinhamento da formação em saúde aos princípios do SUS, para além de iniciativas isoladas, passou a se materializar 
com a homologação das Diretrizes Curriculares Nacionais (DCNs) dos cursos de graduação do campo da saúde, por meio do Parecer no 1.133, emitido em 7 de agosto de 2001 (Brasil, 2001).

Sendo assim, a EIP no Brasil vai ao encontro dos princípios do SUS, especialmente por fomentar a integralidade das ações e dos serviços de saúde, em consonância com a complexidade das necessidades da população em nosso contexto sócio-histórico (Costa, 2016; Peduzzi, 2016). Logo, a EIP tem proporcionado relevantes contribuições em duas vertentes básicas: (1) a melhoria da qualidade da atenção à saúde e (2) a reorganização curricular da formação profissional em saúde, inclusive na pós-graduação. Tais contribuições, a propósito, foram potencializadas pelo plano de cooperação técnica da Organização Pan-Americana de Saúde (OPAS), que lançou, em 2017, a Rede Regional de Educação Interprofissional das Américas (REIP), a qual tem fomentado a interlocução entre organizações governamentais, educacionais e profissionais de 19 países (Gontijo, Freire Filho, \& Forster, 2019).

Deve-se sublinhar que o governo brasileiro, especialmente nas duas últimas décadas, tem investido em iniciativas de EIP, entre as quais a Política Nacional de Educação Permanente em Saúde (PNEPS), de 2004, que instituiu os Programas de Residência em Saúde Uni e Multiprofissional, o Programa Nacional de Reorientação da Formação Profissional em Saúde (Pró-Saúde), de 2005, e o Programa de Educação para o Trabalho em Saúde (PET-Saúde), de 2008 (Dias, Pereira, Batista, \& Casanova, 2016). E cabe mencionar ainda a criação do Plano Nacional para Implementação da Educação Interprofissional, capitaneado pelo Ministério da Educação (MEC) para o biênio 2017-2018 (Costa, Peduzzi, Freire Filho, \& Silva, 2018).

Diante do exposto, é possível afirmar que a EIP vem tendo sua importância reconhecida progressivamente no plano concreto da saúde pública (Freire Filho, Silva, Costa, \& Forster, 2019; Barr, 2015). Contudo, há certas resistências quanto aos seus desdobramentos no país, até mesmo porque a execução de práticas colaborativas encontra uma série de obstáculos em razão da tendência à divisão do trabalho que atravessa modelos de formação e atuação dos profissionais de saúde (Costa, 2016). Alguns caminhos para a superação desses obstáculos foram demarcados por pesquisas sobre EIP realizadas nos últimos anos no Brasil (Peduzzi, Norman, Germani, Silva, \& Souza, 2013). Justifica-se, portanto, um mapeamento do conhecimento disponível a respeito. O presente estudo foi concebido precisamente para tanto, pois teve como objetivo estabelecer um panorama da produção científica nacional relativa à EIP, com ênfase nas pesquisas desenvolvidas com a participação de pós-graduandos ou profissionais de saúde ao longo de uma década (2008-2018).

\section{Método}

Os procedimentos metodológicos típicos das revisões integrativas foram empregados no presente estudo. Tais procedimentos se referem, basicamente: (1) à definição do tema da revisão; (2) à busca e à seleção de referências em bases de dados; (3) à demarcação das informações a serem extraídas das referências; (4) à análise das referências; (5) à síntese dos resultados; e (6) à agregação das evidências científicas disponíveis (Mendes, Silveira, \& Galvão, 2008; Souza, Silva, \& Carvalho, 2010). Vale destacar que as revisões integrativas se afiguram como uma modalidade de revisão da literatura particularmente inclusiva, pois permitem a admissão, no corpus de análise, de publicações derivadas de pesquisas desenvolvidas a partir de diversos delineamentos (Soares et al., 2014). 
No presente estudo, a busca de referências se deu no período compreendido entre 10 de outubro de 2018 e 5 de dezembro de 2018, sendo realizada nas bases de dados Medical Literature Analysis and Retrieval System Online (MEDLINE), Literatura Latino-Americana e do Caribe em Ciências da Saúde (LILACS), Scientific Electronic Library Online-Brasil (SciELOBrasil) e Scopus. Optou-se pelos descritores "educação em pós-graduação", "educação de pós-graduação em saúde", "equipe de assistência em saúde", "aprendizagem interprofissional"; "aprendizagem compartilhada", "aprendizagem colaborativa", "educação interprofissional" e "interprofissional". Tais descritores foram selecionados porque vêm sendo amplamente utilizados em publicações nacionais sobre EIP e se revelaram mais produtivos do que outros semelhantes em buscas prévias, embora alguns deles não constem dos Descritores em Ciências da Saúde (DeCS), vocabulário técnico criado pela Biblioteca Virtual em Saúde (BVS) e adotado por muitos periódicos científicos brasileiros².

Mais especificamente, os descritores foram cruzados como demonstra a Figura 1. No entanto, cabe mencionar que o descritor "educação de pós-graduação em saúde" foi utilizado nas bases de dados SciELO-Brasil e Scopus, devido à natureza generalista delas, sendo substituído por um descritor correspondente, "educação de pós-graduação", nas bases de dados LILACS e MEDLINE, específicas do campo da saúde. Essa estratégia também foi adotada por ter se mostrado mais proveitosa do que outras possíveis em buscas prévias. Com o intuito de viabilizar a localização do maior número de referências possível, os campos de busca utilizados foram os mais abrangentes dentre aqueles existentes em cada uma das bases de dados consultadas: "todos os campos" na SciELO-Brasil e na Scopus, "todos os índices" na LILACS e "título/resumo/assunto" na MEDLINE. Ademais, não foram utilizados quaisquer limites oferecidos pelas bases de dados.

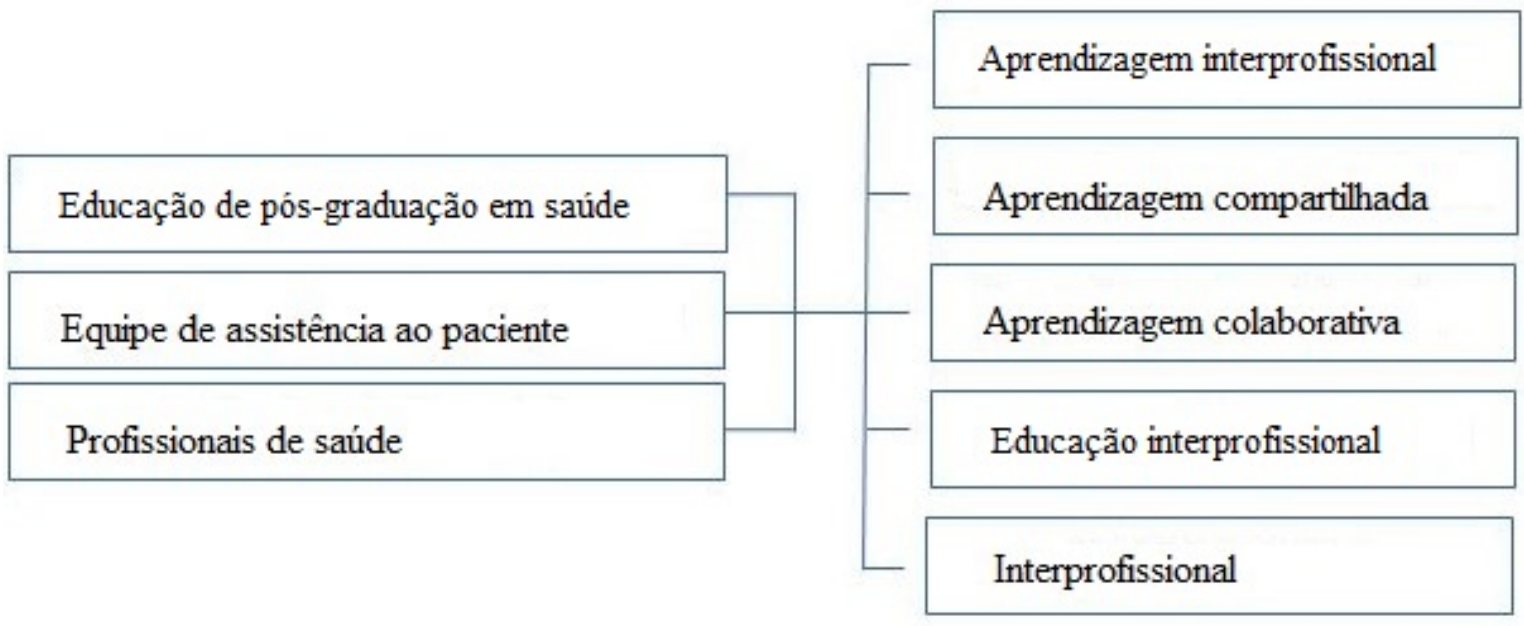

Figura 1 - Cruzamentos dos descritores utilizados

As referências localizadas foram arquivadas no software EndNote Web e passaram por um processo de rastreamento visando ao descarte de eventuais duplicidades. Procedeu-se, então, à leitura dos resumos das referências restantes para determinar o material que viria

\footnotetext{
2 É interessante mencionar que, a despeito de serem frequentemente utilizados como sinônimos, os sufixos "disciplinar" e "profissional" não são equivalentes, pois, em síntese, o primeiro alude ao plano teórico e o segundo ao plano prático (Baquião, 2017). Por essa razão, descritores associados ao sufixo "disciplinar" não foram selecionados para os fins do presente estudo.
} 
a constituir o corpus de análise, levando-se em conta um conjunto de critérios de inclusão. Foram selecionadas as referências: (1) que abordavam temática concernente à EIP junto a pós-graduandos ou profissionais de saúde; (2) que foram publicadas entre 2008 e 2018; (3) decorrentes de pesquisas desenvolvidas no contexto nacional, estando ou não redigidas em português; (4) veiculadas no formato de artigo empírico disponibilizado on-line. Consequentemente, foram excluídas as referências que não se ocupavam diretamente do assunto em pauta, não foram publicadas no período de 10 anos especificado, eram resultantes de pesquisas executadas em outros países ou que se enquadravam em outros formatos de publicação.

A seleção das referências foi empreendida independentemente por dois avaliadores. Discordâncias pontuais foram solucionadas a partir da leitura dos textos completos das referências localizadas e da consulta a um terceiro avaliador. As referências selecionadas foram, então, examinadas, também de modo independente por dois avaliadores, a partir de sete dimensões analíticas estabelecidas, a fim de ordenar o processo de extração de informações, a saber: (1) ano de publicação; (2) autoria e filiação institucional; (3) fonte de publicação; (4) local/região de realização da pesquisa; (5) método; (6) nível de atenção em saúde da pesquisa e (7) principais resultados. Tal exame, cumpre assinalar, foi baseado na leitura dos textos completos das referências selecionadas.

\section{Resultados e Discussão}

As consultas às bases de dados subsidiaram, no total, a localização de 421 referências, sendo 50 na MEDLINE, 90 na LILACS, 143 na SciELO-Brasil e 138 na Scopus. Após a eliminação das duplicidades, contudo, restaram 271 referências, às quais foram aplicados os critérios de inclusão. Como consequência, um número expressivo de referências foi excluído, de modo que o corpus de análise foi constituído por apenas 31 referências. Ressalte-se que, em certo aspecto, já era esperada a exclusão de uma quantidade elevada de referências, em função da abrangência da estratégia de busca empregada. Não obstante, causou certa surpresa o fato de que, em muitas referências localizadas, o termo "interprofissional" foi utilizado de forma pouco criteriosa, sem o estabelecimento de uma articulação mais consistente com a EIP. Logo, o primeiro critério de inclusão foi decisivo para a constituição do corpus de análise.

Em relação à primeira dimensão analítica, verificou-se a seguinte distribuição das referências por ano de publicação: uma em 2010, duas em 2011 e 2013, três em 2014, sete em 2015, seis em 2016, três em 2017 e sete em 2018. Ou seja, é possível observar uma importante concentração de referências (74,19\%) entre 2015 e 2018, o que demonstra que a exploração da EIP na literatura nacional é um fenômeno recente, sobretudo no tocante a pesquisas desenvolvidas junto a pós-graduandos ou profissionais de saúde. E parece razoável reforçar que, acompanhando Michel, Olsson e Toassi (2019) e Peduzzi (2016), o incremento da produção científica consagrada à temática demanda maiores investimentos para impulsionar o estreitamento das relações entre as universidades e os serviços de saúde.

A segunda dimensão analítica revelou que a maioria das referências $(61,29 \%)$ - como aquelas de autoria de Arruda, Barreto, Ribeiro e Frota (2018), Barreto et al. (2018) e Casanova, Batista e Ruiz-Moreno (2015) - foi assinada por membros de diferentes profissões do campo da saúde, o que pode ser considerado positivo, levando-se em conta que a EIP diz respeito à 
formação e à atuação dos profissionais de saúde como um todo. Com relação à filiação institucional, notou-se que todas as referências foram produzidas por pelo menos um pesquisador vinculado a universidades públicas, com destaque para a Universidade de São Paulo (USP) e a Universidade Federal do Ceará (UFC), responsáveis, respectivamente, por 35,48\% e 12,90\% das referências. Tal achado é consistente com aqueles oriundos das análises bibliométricas realizadas por Michel et al. (2019) e Mallmann (2018) sobre a produção científica nacional consagrada à EIP, assim como reflete a tendência histórica à maior produtividade das universidades públicas no Brasil constatada por Sidone, Haddad e Mena-Chalco (2016).

A propósito da terceira dimensão analítica, cabe salientar que as referências selecionadas foram publicadas em 17 periódicos científicos distintos, sendo que a revista Interface - Comunicação, Saúde, Educação concentrou a maior parte delas (29,03\%). A ocorrência de certa diversidade quanto à fonte das referências selecionadas pode ser considerada favorável à difusão da EIP entre membros de diferentes profissões do campo da saúde. Porém, não houve diversidade equivalente no tocante à quarta dimensão analítica. Afinal, verificou-se que a maioria das referências (54,84\%) deriva de pesquisas realizadas na região Sudeste, predominantemente no Estado de São Paulo (32,26\%). Em contrapartida, nenhuma se desenvolveu na região Norte. Tal achado possivelmente está relacionado à notória concentração de cursos de pós-graduação no Sudeste brasileiro, observada por Sidone et al. (2016) e Michel et al. (2019), bem como à existência, nessa região, de grupos de pesquisa que têm se dedicado à EIP, a exemplo daquele intitulado "Gestão e Educação em Saúde e Enfermagem na Perspectiva Colaborativa Interprofissional", abrigado na Escola de Enfermagem da USP.

Os achados referentes à quinta dimensão analítica dão conta de que a maioria das referências $(80,65 \%)$ decorre de pesquisas qualitativas, a exemplo daquelas assinadas por Domingos, Nunes e Carvalho (2015), Ellery, Pontes e Loiola (2013), e Previato e Baldissera (2018). Isso pode ser explicado considerando-se o interesse dos autores em explorar a visão de profissionais de saúde inseridos em instituições de ensino superior ou serviços de saúde quanto a questões relativas à EIP, em especial a execução de práticas colaborativas. É preciso salientar que a definição do método de uma pesquisa se encontra diretamente relacionada ao seu objetivo, de maneira que, como adverte Augusto (2014), não deve ser determinada meramente pela preferência do pesquisador. A autora acrescenta que, por esse motivo, cabe a todo pesquisador renunciar a qualquer tentativa de hierarquização entre os métodos qualitativos e quantitativos.

O nível de atenção em saúde contemplado nas referências selecionadas - sexta dimensão analítica do presente estudo - indica que a atenção primária tem sido privilegiada. Afinal, boa parte delas teve como participantes profissionais de saúde vinculados à Estratégia Saúde da Família (ESF) (22,58\%) ou aos Núcleos de Apoio à Saúde da Família (NASFs) (12,9\%), ou então residentes de Programas de Residência Multiprofissional em Saúde (PRMSs) cuja área de concentração concerne à atenção primária (16,13\%), como se vê nas referências assinadas por Arruda et al. (2018), Faquim e Frazão (2016) e Reis, Medeiros, Pacheco e Caixeta (2016). Tais achados possivelmente espelham o movimento de reorientação do fluxo assistencial do SUS a partir da atenção primária, o qual foi intensificado pela criação do Programa Saúde da Família (PSF), em 1994 (Brasil, 1994). Além disso, os serviços de saúde correspondentes à atenção primária podem ser considerados especialmente compatíveis com as premissas da 
EIP, pois se afiguram como um locus no qual tendem a ser estimulados o trabalho em equipe e o empreendimento de práticas colaborativas (Mallmann, 2018).

A sétima e última dimensão analítica compreende os principais resultados reportados pelas referências selecionadas. Optou-se por organizá-los em eixos temáticos, delimitados de acordo com o foco das referências. Identificou-se que 21 referências $(67,74 \%)$ priorizaram a atuação interprofissional, oito referências $(25,81 \%)$ contemplaram a integração de estratégias de atuação e aprendizagem interprofissional e apenas duas referências (6,45\%) foram dedicadas preponderantemente ao desenvolvimento de estratégias de aprendizagem interprofissional. Ou seja, a maioria das referências tratou de questões relativas ao trabaIho desenvolvido em serviços de saúde na perspectiva da articulação entre as atividades interprofissionais e as atividades específicas de determinadas profissões do campo da saúde, o que reforça a constatação de Peduzzi (2016), de acordo com a qual, no Brasil, observam-se mais iniciativas direcionadas à atuação do que à aprendizagem interprofissional, situação que, ainda para a autora, somente poderá ser revertida com uma inserção mais efetiva da EIP nas grades curriculares dos cursos de graduação em saúde.

Publicações recentes sinalizam que essa inserção vem ocorrendo, por exemplo, na Universidade Federal do Rio Grande do Sul (UFRGS) e na Universidade Federal de São Paulo (UNIFESP), no campus da Baixada Santista. A primeira dessas universidades desenvolve ações de EIP na atenção primária por meio de uma disciplina compartilhada na grade curricular de 15 cursos de graduação em saúde, a qual, a partir de diferentes estratégias de ensino-aprendizagem, prioriza a análise do território e o trabalho em equipe, bem como a aprendizagem interprofissional (Toassi, Olsson, Lewgoy, Bueno, \& Peduzzi, 2020). Já a segunda universidade conta com um projeto pedagógico voltado à interação entre diferentes profissões da saúde, sendo que todos os seus seis cursos são direcionados por três eixos de formação comuns e um eixo específico e, assim, rompem com os modelos tradicionais de ensino centrados nas disciplinas e na formação uniprofissional (Batista et al., 2018).

As referências enquadradas no primeiro eixo temático sublinham a importância, na visão de diferentes profissionais de saúde, das práticas colaborativas para a melhoria da atenção em saúde, mas apontam a existência de obstáculos devido a questões organizacionais e relacionais (Araújo \& Galimbertti, 2013; Barros \& Ellery, 2016; Caram, Rezende, \& Brito, 2017; Souza, Peduzzi, Silva, \& Carvalho; 2016; Faquim \& Frazão, 2016; Goulart, Camelo, Simões, \& Chaves, 2016; Previato \& Baldissera, 2018). A lógica hierárquica e verticalizada ainda presente em muitos serviços de saúde foi apontada como o obstáculo organizacional que mais dificulta a atuação interprofissional, uma vez que, contrariando as premissas da EIP, confere maior relevância a certos saberes em detrimento de outros (Arruda \& Moreira, 2018; Bispo Júnior \& Moreira, 2018; Freire Filho, Costa, Magnago, \& Forster, 2018; Oliveira, Lemes, Machado, Silva, \& Miranda, 2010; Valadão, Lins, \& Carvalho, 2017). Por outro lado, o compartilhamento de responsabilidades foi considerado fundamental para a qualidade do cuidado oferecido aos pacientes (Domingos et al., 2015; Padula \& Aguilar-da-Silva, 2014).

Outro importante obstáculo organizacional à implementação da EIP, segundo os participantes das referências de Arruda e Moreira (2018), Bispo Júnior e Moreira (2018), Caram et al. (2017), Pirolo, Ferraz e Gomes (2011) e Peixoto e Brito (2015), diz respeito à inexistência de dispositivos formais capazes de fomentar a comunicação em muitos serviços de saúde, uma vez que a troca de informações foi reconhecida como fator imprescindível para 
a operacionalização de práticas colaborativas. Constatou-se que, comumente, profissionais de saúde se queixam da falta de horários e espaços físicos regulares para reuniões de equipe (Barros \& Ellery, 2016; Peduzzi, Brígida, Mandú, Souza, \& Silva, 2011; Reis et al., 2016; Rocha, Barreto, \& Moreira, 2016). E, como exposto na referência de autoria de Previato e Baldissera (2018), tal situação pode ser enquadrada de fato como problemática, levando-se em conta que as reuniões de equipe se afiguram como um dispositivo formal potencialmente benéfico à organização dos serviços de saúde.

No que concerne às dificuldades relacionais, as referências assinadas por Arruda e Moreira (2018) e Matuda, Pinto, Martins e Frazão (2015) evidenciam que a forma como se desenvolve a comunicação entre os membros de diferentes profissões do campo da saúde é capaz de comprometer o estabelecimento de relacionamentos mútuos e recíprocos, eventualmente inviabilizando o sentimento de pertencimento à equipe. Como consequência, problemas de convivência podem se mostrar mais frequentes, sendo a fragmentação dos processos de trabalho um de seus possíveis reflexos. Nesse sentido, torna-se patente que as relações interpessoais perpassam o desenvolvimento de práticas colaborativas e, por essa razão, devem ser objeto de reflexão constante em prol da consolidação da EIP (Bispo Júnior \& Moreira, 2018; Ellery et al., 2013; Goulart et al., 2016).

As referências enquadradas no segundo eixo temático lançam luz sobre diferentes questões acerca da integração de estratégias de atuação e aprendizagem interprofissional. Parte dessas referências aponta que, em serviços de saúde nos quais residentes desenvolvem atividades práticas, o trabalho em equipe tende a potencializar o aprendizado mútuo, dialógico e interativo, sobretudo em certas condições: quando são utilizadas metodologias ativas para articular os processos pedagógicos diretamente à resolução de problemas (Araújo, Vasconcelos, Pessoa, \& Forte, 2017), quando os preceptores se encarregam da interlocução junto aos gestores (Arruda et al., 2018), quando é priorizada a construção compartilhada de Projetos Terapêuticos Singulares (PTSs) (Casanova et al., 2015) e quando o cuidado oferecido aos pacientes se beneficia de recursos e equipamentos da comunidade (Gadelha \& Barreto, 2018).

As referências assinadas por Barreto et al. (2018) e Faquim, Buiatti e Frazão (2018), também concernentes ao segundo eixo temático, reportam resultados que dialogam diretamente entre si. Ocorre que a primeira delas, tendo contemplado a ótica de participantes oriundos tanto do campo da saúde quanto do campo da educação, indica que a gestão participativa amplia as possibilidades de aprendizagem no âmbito das parcerias firmadas entre instituições de ensino superior e sistemas municipais de saúde. E, por meio da segunda referência, concluiu-se que o emprego de um método participativo - denominado Método de Planejamento de Projeto Orientado por Objetivos - foi capaz de intensificar a disposição para a colaboração interprofissional em dois serviços de atenção primária, na medida em que o envolvimento dos pacientes no planejamento das intervenções que seriam desenvolvidas junto a eles reforçou compromissos éticos dos profissionais de saúde.

Já Silva, Peduzzi, Orchard e Leonello (2015) constataram que, segundo professores universitários do campo da saúde, a aprendizagem interprofissional passa necessariamente pela valorização de saberes e práticas que se concretiza desde a discussão de casos clínicos até a realização de consultas conjuntas. E, de forma semelhante, Vasconcelos, Stedefeldt e Frutuoso (2016) defenderam que a associação ensino-serviço - conforme membros de 
diferentes profissões do campo da saúde - pode ser equiparada a uma simbiose, por proporcionar importantes vantagens para ambas as partes, contanto que os envolvidos estejam abertos para tanto. Sendo assim, as referências enquadradas no segundo eixo temático reforçam que a inserção acadêmica em serviços de saúde pode culminar na operacionalização de um novo modo de produzir saúde e, para além disso, corroboram as premissas de Reeves (2016), de acordo com as quais métodos de aprendizagem que permitem maior interatividade - e, como consequência, ensejam a superação de modelos de formação tradicionais - são os mais propícios à EIP.

Por fim, os resultados reportados por Perego e Batista (2016) e Figueiredo e Campos (2014) nas duas referências enquadradas no terceiro eixo temático - ou seja, aquelas que se debruçaram especificamente sobre o desenvolvimento de estratégias de aprendizagem interprofissional - sublinham que a pós-graduação pode, de diferentes maneiras, viabilizar a aprendizagem interprofissional. A primeira dessas referências investigou a percepção de residentes, preceptores e tutores sobre a formação para o trabalho em equipe. O PRMS ao qual tais participantes se encontravam vinculados foi reconhecido como um espaço favorável à interprofissionalidade, por priorizar a integração das ações e dos saberes dos diferentes profissionais de saúde e, assim, fomentar a interdependência profissional. Tais achados ilustram que, como observaram Lewgoy, Kuplich, Machado, Faber e Fagundes (2019), as proposições dos PRMSs - como modalidade de pós-graduação lato sensu norteada pela formação em serviço - estão alinhadas às premissas da EIP.

Na segunda e última referência concernente ao terceiro eixo temático, Figueiredo e Campos (2014) avaliaram a utilização do apoio Paideia como referencial pedagógico em um curso de especialização. Trata-se de um recurso conceitual e metodológico que parte de uma crítica à racionalidade gerencial hegemônica e visa a favorecer a gestão democrática em contextos institucionais e a ampliar as capacidades de análise e tomada de decisões por parte de gestores, trabalhadores e também usuários (Campos, 2000). Os autores da referência em pauta verificaram que os participantes - membros de diferentes profissões do campo da saúde - encontraram no curso um espaço coletivo em que, especialmente a partir da discussão de casos clínicos, puderam aprimorar competências técnicas, éticas e relacionais (Figueiredo \& Campos, 2014). Portanto, evidenciou-se que em cursos de especialização, também, diferentes profissionais de saúde podem aprender juntos para que venham a trabalhar, em conjunto, de maneira mais qualificada. Essa é uma constatação importante, tendo em vista que, no âmbito de cursos de especialização no país, tende ainda a prevalecer a perspectiva uniprofissional.

\section{Considerações Finais}

O presente estudo, por meio de uma revisão integrativa da literatura, buscou mapear o conhecimento estabelecido sobre a EIP no Brasil, especificamente no tocante às pesquisas que contaram com a participação de pós-graduandos e profissionais de saúde ao longo de uma década (2008-2018). A síntese dos resultados concernentes às dimensões analíticas empregadas demonstra que o interesse pelo tema na produção científica nacional é recente, mas tem aumentado consideravelmente, sobretudo devido ao trabalho de membros de diferentes profissões do campo da saúde, predominantemente vinculados a universidades públicas. 
Constatou-se também que as fontes de publicação são diversificadas, sendo que a maioria das referências selecionadas decorre de pesquisas cujo cenário foi a região Sudeste e nas quais foram privilegiados métodos qualitativos e serviços de saúde concernentes à atenção primária.

Ressalte-se que as evidências científicas proporcionadas pelas referências selecionadas salientam que o fortalecimento da EIP no país se afigura como um importante caminho a ser percorrido no sentido da integralidade das ações e dos serviços de saúde, condição necessária em face das necessidades da população, cada vez mais complexas e dinâmicas no campo da saúde. As referências selecionadas forneceram ainda informações importantes acerca do movimento de reorientação da prática em saúde mediante a valorização do trabalho em equipe no país, e, mais pontualmente, apresentaram indicações para o fomento da aprendizagem interprofissional no âmbito da pós-graduação. Por outro lado, observou-se que uma série de questões segue em aberto. Talvez a principal delas seja a seguinte: como operacionalizar as mudanças culturais - de modo a equacionar as relações de poder no campo da saúde, por exemplo - que se fazem necessárias para que o empreendimento de práticas colaborativas seja sustentável em longo prazo no país?

Novas pesquisas certamente auxiliarão a construir respostas para essa questão, particularmente se, de acordo com o panorama delineado no presente estudo, conferirem maior ênfase à exploração de estratégias de aprendizagem interprofissional. Não obstante, é preciso mencionar que o presente estudo tem um alcance determinado pelo fato de ter se ocupado exclusivamente de referências veiculadas no formato de artigo. Com isso, não foram contempladas teses e dissertações, por exemplo. Referências veiculadas nos formatos em questão poderiam ampliar o corpus de análise. Contudo, revisões integrativas comumente privilegiam artigos, por se tratar de literatura revisada e publicada de acordo com as práticas mais valorizadas pela comunidade científica.

\section{Referências}

Araújo, E. M. D., \& Galimbertti, P. A. (2013). A colaboração interprofissional na estratégia saúde da família. Psicologia \& Sociedade, 25(2), 461-468. Recuperado de http://www.scielo.br/ scielo.php?script=sci_arttext\&pid=S0102-71822013000200023\&lng=en\&tlng=pt

Araújo, T. A. M., Vasconcelos, A. C. C. P., Pessoa, T. R. R. F., \& Forte, F. D. S. (2017). Multiprofissionalidade e interprofissionalidade em uma residência hospitalar: O olhar de residentes e preceptores. Interface - Comunicação, Saúde, Educação, 21(62), 601-613. doi:http://dx.doi.org/10.1590/1807-57622016.0295

Arruda, G. M. M. S., Barreto, I. C. H. C., Ribeiro, K. G., \& Frota, A. C. (2018). O desenvolvimento da colaboração interprofissional em diferentes contextos de residência multiprofissional em Saúde da Família. Interface - Comunicação, Saúde, Educação, 22(supl. 1), 1309-23. doi:http://dx.doi.org/10.1590/1807-57622016.0859

Arruda, L. S., \& Moreira, C. O. F. (2018). Colaboração interprofissional: Um estudo de caso sobre os profissionais do Núcleo de Atenção ao Idoso da Universidade Estadual do Rio de Janeiro (NAI/UERJ), Brasil. Interface - Comunicação, Saúde, Educação, 22(64), 199-210. doi:http://dx.doi.org/10.1590/1807-57622016.0613

Augusto, A. (2014). Metodologias quantitativas/metodologias qualitativas: Mais do que uma questão de preferência. Fórum Sociológico, 24, 73-77. Recuperado de https://journals. openedition.org/sociologico/1073 
Baquião, A. P. S. S. (2017). Percepções de residentes multiprofissionais acerca da interdisciplinaridade (Dissertação de Mestrado, Instituto de Ciências Humanas Universidade Federal de Juiz de Fora). Recuperado de http://repositorio.ufjf.br:8080/jspui/handle/ufjf/6489

Barr, H. (2015). Interprofessional education: The genesis of a global movement. Farenham: Centre for the Advancement of Interprofessional Education. Recuperado de https://www.caipe.org/ resources/publications/barr-h-2015-interprofessional-education-genesis-global-movement

Barr, H., \& Low, H. (2013). Introducing Interprofessional Education. Fareham: Centre for the Advancement of Interprofessional Education. Recuperado de https://www. caipe.org/resources/publications/caipe-publications/barr-h-low-h-2013-introducinginterprofessional-education-13th-november-2016

Barreto, I. C. H. C., Ribeiro, K. G., Moreira, A. E. M. M., Goya, N., Dias, M. S. A., \& Andrade, L. O. M. (2018). Integração de instituições de ensino superior com sistemas municipais de saúde à luz de uma tipologia da colaboração interprofissional. Interface - Comunicação, Saúde, Educação, 22(1), 1365-1376. doi:http://dx.doi.org/10.1590/1807-57622016.0860

Barros, E. R. S., \& Ellery, A. E. L. (2016). Colaboração interprofissional em uma Unidade de Terapia Intensiva: Desafios e possibilidades. Revista da Rede de Enfermagem do Nordeste, 17(1), 10-19. doi:http://dx.doi.org/10.15253/2175-6783.2016000100003

Batista, N. A., Rossit, R. A. S., Batista, S. H. S. D. S., Silva, C. C. B. D., Uchôa-Figueiredo, L. D. R., \& Poletto, P. R. (2018). Educação interprofissional na formação em Saúde: A experiência da Universidade Federal de São Paulo, campus Baixada Santista, Santos, Brasil. Interface - Comunicação, Saúde, Educação, 22(Supl. 2), 1705-1715. doi:http:// dx.doi.org/10.1590/1807-57622017.0693

Bispo Júnior, J. P., \& Moreira, D. C. (2018). Cuidado colaborativo entre os Núcleos de Apoio à Saúde da Família e as equipes apoiadas. Physis: Revista de Saúde Coletiva, 28(3), e280310. doi: http://dx.doi.org/10.1590/s0103-73312018280310

Blue, A., Brandt, B. F., \& Schmitt, M. H. (2010). American Interprofessional Health Collaborative: Historical roots and organizational beginnings. Journal of Allied Health, 39(suppl. 1), 204209. Recuperado de https://www.ncbi.nIm.nih.gov/pubmed/21174041?dopt=Abstract

Brasil. (2001, 3 de outubro). Ministério da Educação e Cultura. Câmara de Educação Superior. Parecer CNE/CES n. 1133/2001. Diretrizes Curriculares Nacionais dos Cursos de Graduação em Enfermagem, Medicina e Nutrição. Diário Oficial da União, Seção 1E, p. 131. Brasília-DF: Ministério da Educação e Cultura. Recuperado de http://portal.mec.gov. br/dmdocuments/ces1133.pdf

Brasil. (1994). Ministério da Saúde. Programa Saúde da Família. Brasília. Brasília-DF: COSAC. Campos, G. W. S. (2000). Um método para análise e cogestão de coletivos. São Paulo: Hucitec. Canadian Interprofessional Health Collaborative. (2010). A national interprofessional competency framework. Vancouver: CIHC. Recuperado de https://www.cihc.ca/files/ CIHC_IPCompetencies_Feb1210.pdf

Caram, C. D. S., Rezende, L. C., \& Brito, M. J. M. (2017). Prática colaborativa: Potencialidades e desafios para o enfermeiro no contexto hospitalar. Revista Mineira de Enfermagem, 21, e1070. doi:http://dx.doi.org/10.5935/1415-2762.20170080

Casanova, I. A., Batista, N. A., \& Ruiz-Moreno L. (2015). Formação para o trabalho em equipe na residência multiprofissional em saúde. ABCS Health Sciences, 40(3), 229-233. doi:http://dx.doi.org/10.7322/abcshs.v40i3.800 
Costa, M. V. D. (2016). A educação interprofissional no contexto brasileiro: Algumas reflexões. Interface - Comunicação, Saúde, Educação, 20(56), 197-198. doi:http://dx.doi. org/10.1590/1807-57622015.0311

Costa, M. V. D., Peduzzi, M., Freire Filho, J. R., \& Silva, C. B. G. (2018). Educação interprofissional em saúde. Natal: Secretaria de Educação a Distância/Universidade Federal do Rio Grande do Norte. Recuperado de https://portalarquivos2.saude.gov.br/images/pdf/2018/ dezembro/12/Educacao-Interprofissional-em-Saude.pdf

Dias, I. M. A. V., Pereira, A. K., Batista, S. H. S. S., \& Casanova, I. A. (2016). A tutoria no processo de ensino-aprendizagem no contexto da formação interprofissional em saúde. Saúde em Debate, 40(111), 257-267. doi: http://dx.doi.org/10.1590/0103-1104201611120

Domingos, C. M., Nunes, E. F. P. A., \& Carvalho, B. G. (2015). Potencialidades da Residência Multiprofissional em Saúde da Família: o olhar do trabalhador de saúde. Interface - Comunicação, Saúde, Educação, 19(55), 1221-1232. doi: http://dx.doi. org/10.1590/1807-57622014.0653

Ellery, A. E. L., Pontes, R. J. S., \& Loiola, F. A. (2013). Campo comum de atuação dos profissionais da Estratégia Saúde da Família no Brasil: Um cenário em construção. Physis: Revista de Saúde Coletiva, 23(2), 415-437. doi: http://dx.doi.org/10.1590/S0103-73312013000200006

Faquim, J. P. S., \& Frazão, P. (2016). Percepções e atitudes sobre relações interprofissionais na assistência odontológica durante o pré-natal. Saúde em Debate, 40(109), 59-69. doi: http://dx.doi.org/10.1590/0103-1104201610905

Faquim, J. P. S., Buiatti, N. B. P., \& Frazão, P. (2018). O método ZOPP e a organização do trabalho interprofissional voltado à atenção ao pré-natal em duas unidades de atenção básica. Saúde em Debate, 42(117), 392-407. doi: http://dx.doi.org/10.1590/0103-1104201811704

Feuerwerker, L. C. M., \& Capozzolo, A. A. (2013). Mudanças na formação dos profissionais de saúde: alguns referenciais de partida do eixo trabalho em saúde. In A. A. Capozzolo, S. J., Casetto, \& A. O. Hens (Org.), Clínica comum: Itinerários de uma formação em saúde (pp. 35-68). São Paulo: Hucitec.

Figueiredo, M. D., \& Campos, G. W. S. (2014). O apoio Paideia como metodologia para processos de formação em saúde. Interface - Comunicação, Saúde, Educação, 18(1), 931943. doi:http://dx.doi.org/10.1590/1807-57622013.0323

Freire Filho, J. R., Costa, M. V., Magnago, C., \& Forster, A. C. (2018). Atitudes para a colaboração interprofissional de equipes da Atenção Primária participantes do Programa Mais Médicos. Revista Latino-Americana de Enfermagem, 26, e3018. doi:http://dx.doi. org/10.1590/1518-8345.2731.3018

Freire Filho, J. R., Silva, C. B. G., Costa, M. V., \& Forster, A. C. (2019). Educação Interprofissional nas políticas de reorientação da formação profissional em saúde no Brasil. Saúde em Debate, 43(supl. 1), 86-96.

Gadelha, A. K. S., \& Barreto, I. C. H. C. (2018). Residência integrada em Saúde: Percepção dos atores da ênfase em Saúde da Família e Comunidade. Interface - Comunicação, Saúde, Educação, 22(1), 1339-1351. doi:http://dx.doi.org/10.1590/1807-57622017.0183

Gontijo, E. D., Freire Filho, J. R., \& Forster, A. C. (2019). Educação Interprofissional em Saúde: abordagem na perspectiva de recomendações internacionais. Cadernos do Cuidado, 3(2), 20-38. doi:http://dx.doi.org/10.29397/cc.v3n2.186 
Goulart, B. F., Camelo, S. H. H., Simões, A. L. A., \& Chaves, L. D. P. (2016). Trabalho em equipe em Unidade Coronariana: Facilidades e dificuldades. Revista da Escola de Enfermagem da USP, 50(3), 482-489. doi:http://dx.doi.org/10.1590/S0080-623420160000400015

Kantrowitz, M., Kaufman, A., Mennin, S., Fülöp, T. A., \& Guilbert, J. J. (1987). Innovative tracks at established institutions for the education of health personnel: An experimental approach to change relevant to health needs. Genebra: World Health Organization. Recuperado de https://apps.who.int/iris/bitstream/handle/10665/38557/WHO_OFFSET_101_Partl-II. pdf?sequence $=1 \&$ isAllowed $=y$

Lewgoy, A. M. B., Kuplich, N. M., Machado, R. N., Faber, T., \& Fagundes, M. L. (2019). A perspectiva interprofissional na residência integrada multiprofissional em saúde hospitalar. Clinical \& Biomedical Research, 39(1), 58-68. Recuperado de https://seer.ufrgs.br/hcpa/ article/view/87747/pdf

Mallmann, F. H. (2018). Educação e trabalho interprofissional em saúde no contexto da atenção primária no Brasil: Análise bibliométrica da produção científica de 2010 a 2017 (Trabalho de Conclusão de Curso, Especialização em Saúde Pública, Faculdade de Medicina, Universidade Federal do Rio Grande do Sul). Recuperado de https://www.lume. ufrgs.br/bitstream/handle/10183/184287/001077855.pdf?sequence=1\&isAllowed=y

Matuda, C. G., Pinto, N. R. S., Martins, C. L., \& Frazão, P. (2015). Colaboração interprofissional na Estratégia Saúde da Família: Implicações para a produção do cuidado e a gestão do trabalho. Ciência \& Saúde Coletiva, 20(8), 2511-2521. doi:http://dx.doi. org/10.1590/1413-81232015208.11652014

Mendes, K. D. S., Silveira, R. C. C. P., \& Galvão, C. M. (2008). Revisão integrativa: método de pesquisa para a incorporação de evidências na saúde e na enfermagem. Texto \& ContextoEnfermagem, 17(4), 758-764. doi: http://dx.doi.org/10.1590/S0104-07072008000400018

Michel, C., Olsson, T. O., \& Toassi, R. F. C. (2019). Educação Interprofissional em Saúde: Análise bibliométrica da produção científica nacional. Revista da Associação Brasileira de Ensino Odontológico, 19(4), 78-90. doi:http://dx.doi.org/10.30979/rev.abeno.v19i4.781

Oliveira, A. M. D., Lemes, A. M., Machado, C. R., Silva, F. L., \& Miranda, F. S. (2010). Relação entre enfermeiros e médicos em hospital escola: a perspectiva dos médicos. Revista Brasileira de Saúde Materno Infantil, 10, 433-439. doi:http://dx.doi.org/10.1590/ S1519-38292010000600023

Organização Mundial de Saúde (2010). Marco para ação em Educação Interprofissional e prática colaborativa. Genebra: World Health Organization. Recuperado de http://www. fnepas.org.br/oms_traduzido_2010.pdf

Padula, M. G. C., \& Aguilar-da-Silva, R. H. (2014). Professional profile of dentists who are members of the Family Health Strategy city of Marilia, São Paulo: The challenge of interprofessional work. Revista de Odontologia da UNESP, 43(1), 52-60. doi:http://dx.doi. org/10.1590/S1807-25772014000100009

Peduzzi, M. (2016). O SUS é interprofissional. Interface - Comunicação, Saúde, Educação, 20(56), 199-201. doi:http://dx.doi.org/10.1590/1807-57622015.0383

Peduzzi, M., Norman, I. J., Germani, A. C. C. G., Silva, J. A. M., \& Souza, G. C. (2013). Educação interprofissional: formação de profissionais de saúde para o trabalho em equipe com foco nos usuários. Revista da Escola de Enfermagem da USP, 47(4), 977-983. doi:http://dx.doi. org/10.1590/S0080-623420130000400029 
Peduzzi, M., Brígida, G. C., Mandú, E. N. T., Souza, G. C., \& Silva, J. A. M. (2011). Trabalho em equipe na perspectiva da gerência de serviços de saúde: Instrumentos para a construção da prática interprofissional. Physis: Revista de Saúde Coletiva, 21(2), 629-646. doi:http:// dx.doi.org/10.1590/s0103-73312011000200015

Peixoto, T. C., \& Brito, M. J. M. (2015). Protocolo clínico como dispositivo analítico das relações de poder de profissionais de saúde. Saúde em Debate, 39(107), 1053-1064. doi:http:// dx.doi.org/10.1590/0103-110420151070219

Perego, M. G., \& Batista, N. A. (2016). Aprendizagens compartilhadas na Residência Multiprofissional em Saúde. Tempus: Actas de Saúde Coletiva, 10(4), 39-51. Recuperado de http://www.tempusactas.unb.br/index.php/tempus/article/view/2239/1708

Pirolo, S. M., Ferraz, C. A., \& Gomes R. (2011). A integralidade do cuidado e ação comunicativa na prática interprofissional da terapia intensiva. Revista da Escola de Enfermagem da USP, 45(6), 1396-1402. doi:http://dx.doi.org/10.1590/S0080-62342011000600017

Previato, G. F., \& Baldissera, V. D. A. (2018). Retratos da prática interprofissional colaborativa nas equipes da atenção primária à saúde. Revista Gaúcha de Enfermagem, 39, e20170132. doi:http://dx.doi.org/10.1590/1983-1447.2018.2017-0132

Reeves, S. (2016). Porque precisamos da educação interprofissional para um cuidado efetivo e seguro. Interface - Comunicação, Saúde, Educação, 20(56), 185-197. doi:http://dx.doi. org/10.1590/1807-57622014.0092

Reeves, S., Abramivich I., \& Goldman J. (2009). Canadian Interprofessional Health Collaborative activities, outputs and impacts evaluation. Vancouver: Canadian Interprofessional Health Collaborative. Recuperado de http://www.cihc.ca/files/publications/CIHC_ EvalReport0809_May1109.pdf

Reis, M. L., Medeiros, M., Pacheco, L. R., \& Caixeta, C. C. (2016). Avaliação do trabalho multiprofissional do Núcleo de Apoio à Saúde da Família (NASF). Texto \& Contexto Enfermagem, 25(1), 1-9. doi:http://dx.doi.org/10.1590/0104-070720160002810014

Rocha, F. A. A., Barreto, I. C. H. C., \& Moreira, A. E. M. M. (2016). Colaboração interprofissional: Estudo de caso entre gestores, docentes e profissionais de saúde da família. Interface - Comunicação, Saúde, Educação, 20(57), 415-426. doi:http://dx.doi. org/10.1590/1807-57622015.0370

Sidone, O. J. G., Haddad, E. A., \& Mena-Chalco, J. P. (2016). A ciência nas regiões brasileiras: Evolução da produção e das redes de colaboração científica. Transinformação, 28(1), 15 32. doi:http://dx.doi.org/10.1590/2318-08892016002800002

Silva, J. A. M., Peduzzi, M., Orchard, C., \& Leonello, V. M. (2015). Educação interprofissional e prática colaborativa na Atenção Primária à Saúde. Revista da Escola de Enfermagem da USP, 49(2), 16-24. doi:http://dx.doi.org/10.1590/S0080-623420150000800003

Soares, C. B., Hoga, L. A. K., Peduzzi, M., Sangaleti, C., Yonekura, T., \& Silva, D. R. A. D. (2014). Revisão integrativa: Conceitos e métodos utilizados na enfermagem. Revista da Escola de Enfermagem da USP, 48(2), 335-345. doi:http://dx.doi.org/10.1590/ S0080-6234201400002000020

Souza, G. C., Peduzzi, M., Silva, J. A. M., \& Carvalho, B. G. (2016). Trabalho em equipe de enfermagem: Circunscrito à profissão ou colaboração interprofissional? Revista da Escola de Enfermagem da USP, 50(4), 642-649. doi:http://dx.doi.org/10.1590/ S0080-623420160000500015 
Souza, M. T., Silva, M. D., \& Carvalho, R. (2010). Revisão integrativa: O que é e como fazer. Einstein, 8(1), 102-106. doi: http://dx.doi.org/10.1590/s1679-45082010rw1134

Toassi, R. F. C., Olsson, T. O., Lewgoy, A. M. B., Bueno, D., \& Peduzzi, M. (2020). Ensino da graduação em cenários da atenção primária: Espaço para aprendizagem interprofissional. Trabalho, Educação e Saúde, 18(2). doi:http://dx.doi.org/10.1590/1981-7746-sol00267

Valadão, P. A. S., Lins, L., \& Carvalho, F. M. (2017). Problemas bioéticos no cotidiano do trabalho de profissionais de equipes de saúde da família. Trabalho, Educação e Saúde, 15(3), 725-744. doi:http://dx.doi.org/10.1590/1981-7746-sol00080

Vasconcelos, A. C. F., Stedefeldt, E., \& Frutuoso, M. F. P. (2016). Uma experiência de integração ensino-serviço e a mudança de práticas profissionais: Com a palavra, os profissionais de saúde. Interface - Comunicação, Saúde, Educação, 20(56), 147-158. doi:http://dx.doi. org/10.1590/1807-57622015.0395

Recebido em: 25/03/2018

Última revisão: 10/12/2020

Aceite final: 12/01/2021

\section{Sobre os autores:}

Ana Paula de Sousa Silva Baquião: Doutoranda do Programa de Pós-Graduação em Psicologia da Universidade Federal de Juiz de Fora (UFJF). E-mail: anapaulasousa.psico@gmail.com, Orcid: https://orcid.org/0000-0002-5300-9397

Bruna Costa de Almeida: Graduanda em Psicologia pela Universidade Federal de Juiz de Fora (UFJF). E-mail: bnacalmeida@gmail.com, Orcid: https://orcid.org/0000-0002-4279-28572957-7554

Leila Garcia Ribeiro Silva: Graduanda em Psicologia pela Universidade Federal de Juiz de Fora (UFJF). E-mail: leilariibeiro@gmail.com, Orcid: https://orcid.org/0000-0003-0172-9357

Rodrigo Sanches Peres: Doutor e mestre em Psicologia pela Universidade de São Paulo (USP). Professor associado do Instituto de Psicologia da Universidade Federal de Uberlândia (UFU). Bolsista de Produtividade em Pesquisa do Conselho Nacional de Desenvolvimento Científico e Tecnológico (CNPq). E-mail: rodrigosanchesperes@ufu.br, Orcid: https://orcid.org/0000-0002-2957-7554

Fabiane Rossi dos Santos Grincenkov: Doutora e mestre em Saúde pela Universidade Federal de Juiz de Fora (UFJF). Professora adjunta do Departamento de Psicologia da UFJF. E-mail: fabiane.rossi@ufjf.edu.br, Orcid: https://orcid.org/0000-0003-0948-2635 
